\title{
Toward 10TB Cartridge Capacity with Magneto-Optical Recording on Ultra Thin Discs
}

\author{
M. Tani \\ Development \& Technology Division, Hitachi Maxell, Ltd., 6-20-1 Kinunodai, Tsukubamirai-shi, Ibaraki, 300-2496 Japan
}

\begin{abstract}
Magneto-Optical (MO) recording on ultra thin discs with $92 \mu \mathrm{m}$ thickness succeeded. Key technologies are a nano-imprint for embossing on the thin disc and aerodynamic stabilizer (ADS) for stable disc rotation. The ADS made the thin disc rotation stable by providing an air cushion between the glass stabilizer and thin disc. The ADS was also available for the thin disc with a magnetic flying head. Using TbFeCo film, the thin disc had almost the same performance as that of a normal disc with $1.2 \mathrm{~mm}$ thickness. Stacked Volumetric Optical Discs (SVOD) can enhance the volumetric capacity with a lot of thin discs stored in a compact cartridge. Because the $40 \mathrm{~nm}$ mark length recording corresponds to the $100 \mathrm{~GB} /$ disc with $\mathrm{CD}$ size which has already succeeded in MO recording, the combination of MO recording and SVOD with 100 thin discs will enable the possibility to provide a $10 \mathrm{~TB}$ cartridge capacity.
\end{abstract}

Key words: Magneto-Optical recording, nano-imprint, thin optical disc, SVOD

\section{Introduction}

The capacity of storage media has increased with improvements in areal recording density. In the pursuit of ultra high density recording, Magneto-Optical (MO) recording has shown itself to be a very strong candidate, because it is possible to obtain signal from smaller domains under optical limitation by using Magnetically induced Super Resolution $(\mathrm{MSR})^{1}$. We have already reached the $40 \mathrm{~nm}$ mark length recording with Magnetic Amplifying Magneto-optical System (MAMMOS) ${ }^{2}$ as shown in Figure $1^{3)}$. This corresponds to a $100 \mathrm{~GB} /$ disc with $\mathrm{CD}$ size.

On the other hand, Stacked Volumetric Optical Discs (SVOD) ${ }^{4)}$ has been reported as the technology to enhance volumetric capacity with thin optical discs. Thin discs less than $0.1 \mathrm{~mm}$ thickness have 10 times the data capacity per volume of normal optical discs with $1.2 \mathrm{~mm}$ thickness. 100 thin discs stored in a compact cartridge. A disc changer for thin discs allows us to handle 100 times data capacity than that of a conventional optical disc ${ }^{5)}$.

The combination of MO recording and SVOD gives us the possibility of providing a $10 \mathrm{~TB}$ cartridge capacity. This paper describes the basic characteristics of thermally assisted recording on the thin discs with $92 \mu \mathrm{m}$ thickness.

\section{Experimental Procedure}

\subsection{Thin disc structure}

Figure 2 shows a photograph of a thin disc. The thin disc is so flexible that it almost flutters in a breath of air.

The substrate of the thin disc is a polycarbonate (PC) film with $92 \mu \mathrm{m}$ thickness. The groove structure is formed on its surface by nano-imprint technology6). The nano-imprint procedure is as follows. The PC film is placed between a base plate and stamper. They are heated and pressed. The operation temperature during pressing is above the glass transition temperature $(\mathrm{Tg})$ of the PC film corresponds to 145 degrees Celsius. The PC film is released from the stamper after the cooling process. Nano-imprint can be completed in 8 seconds. This value is close to a conventional injection mold. Figure 3 shows an AFM image of the surface on the PC film after nano-imprint. The track pitch is $0.74 \mu \mathrm{m}$ consisting of groove width, $0.42 \mu \mathrm{m}$. The groove structure formed by nano-imprint is as good as that of an injection mold.

The disc structure for thermally assisted magnetic recording is UV resin $(5 \mu \mathrm{m}) / \mathrm{Ag}$ alloy $(20 \mathrm{~nm}) / \mathrm{SiN}(5$ $\mathrm{nm}) / \mathrm{TbFeCo}(40 \mathrm{~nm}) / \mathrm{SiN}(60 \mathrm{~nm}) / \mathrm{PC}$ substrate(92 $\mu \mathrm{m})$. Each of the layers was prepared by a magnetron sputtering process. The UV resin was prepared by a spin coat method.

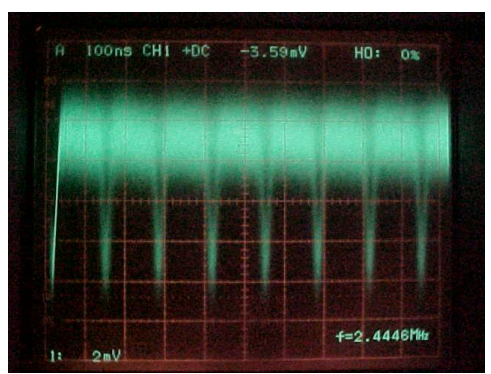

Fig. 1 Readout signal for 40nm mark length with $1.2 \mathrm{~mm}$ thick substrate.

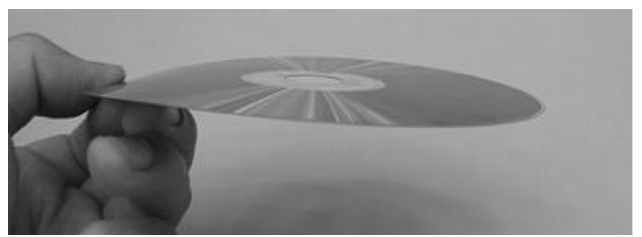

Fig. 2 Photograph of a thin disc. 


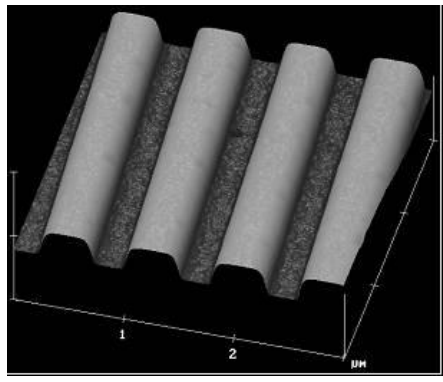

Fig. 3 AFM image of the surface on PC film after nano-imprint.

\subsection{Experimental setup}

The write and read operation for the ultra thin disc requires a stable disc rotation. We developed an aerodynamic stabilizer (ADS) for the stable rotation of the thin disc. Figure 4 shows a cross section view of the experimental setup with ADS.

A glass substrate with $0.5 \mathrm{~mm}$ thickness is attached on the top of a spindle motor. A spacer of which thickness, inside and outside diameter are 0.2 $\mathrm{mm}, \varphi 15 \mathrm{~mm}$ and $\varphi 25 \mathrm{~mm}$ is placed on the center of the glass substrate. In order to produce an aerodynamic effect on the thin disc, the glass substrate has 8 holes just outside the spacer, centrosymmetrically. The diameter of each hole is $\varphi 6$ $\mathrm{mm}$. The thin disc is set on the spacer and fixed by a clamper. The thin disc rotates with the glass substrate.

When the spindle motor starts to rotate, air flows continually thorough the holes over the glass substrate and passes though the space between the glass substrate and the thin disc due to centrifugal forces. This air current cushions the thin disc on the glass disc surface.

A laser beam irradiated from an optical head goes to the thin disc though the glass substrate. The total thickness from the entrance plane to the recording layer is $0.6 \mathrm{~mm}$. In this work, a magneto-optical (MO) disc tester with a wave length, $650 \mathrm{~nm}$ and an objective lens with numerical aperture, 0.6 was used to write and read. A magnetic flying head for recording is loaded on the thin disc after rotation. Its flying height is $5 \mu \mathrm{m}$ at linear velocity of $5 \mathrm{~m} / \mathrm{s}$ for default readout. The coil generates a 240 Oe magnetic field on the recording layer under a $300 \mathrm{~mA}$ current. Recording is performed by a Laser Pumped Magnetic Field Modulation method (LP-MFM) with a duty of $35 \%$. The read and write area is only in-groove.

\section{Results}

\subsection{Stable rotation of thin disc}

Figure 5 shows the axial run out of the thin disc for one rotation at the linear velocity, $5 \mathrm{~m} / \mathrm{s}$ and the radius, $30 \mathrm{~mm}$. Figure 5 (a) is used ADS as shown in Fig. 4. Figure 5 (b) is used ADS without through holes on the glass substrate. The shape of the axial run out in Fig 5 (a) is almost the same as that of the glass substrate, because the air current cushions the thin disc on the glass disc surface. On the other hand, in the case of no though holes on the glass substrate, a jagged curve appears in Fig. 5 (b), because the air stuck between thin disc and the glass substrate deforms the thin disc unevenly. This partial deformation on the thin disc must disturb focusing. In this way, the aero dynamic effect is very important for stable rotation of the thin disc.

Figure 6 shows a focusing error signal (FES) and tracking error signal (TES) of the thin disc at the linear velocity, $5 \mathrm{~m} / \mathrm{s}$ and the radius, $30 \mathrm{~mm}$ using ADS with through holes on the glass substrate. The FES and TES are stable enough to write and read. These signals did not change the magnetic flying head whether loaded on the thin disc or not. The flying head is adaptable to the thin disc with the ADS.

Figure 7 shows the linear velocity dependence of the maximum amplitude of FES with the magnetic flying head at the radius of $30 \mathrm{~mm}$. (a) $1.2 \mathrm{~mm}$ thick disc, (b) $92 \mu \mathrm{m}$ thick disc with the ADS. The magnetic flying head was made to contact the disc less than the linear velocity of $1.2 \mathrm{~m} / \mathrm{s}$. The FES of the $1.2 \mathrm{~mm}$ thick disc does not change irrespective of the linear velocity. On the other hand, that of the thin disc rises less than linear velocity of $1.2 \mathrm{~m} / \mathrm{s}$, because the friction between the magnetic flying head and thin disc creates a vibration on the thin disc. It is important for the stable rotation of the thin disc with the magnetic flying head that the magnetic flying head does not contact the thin disc.

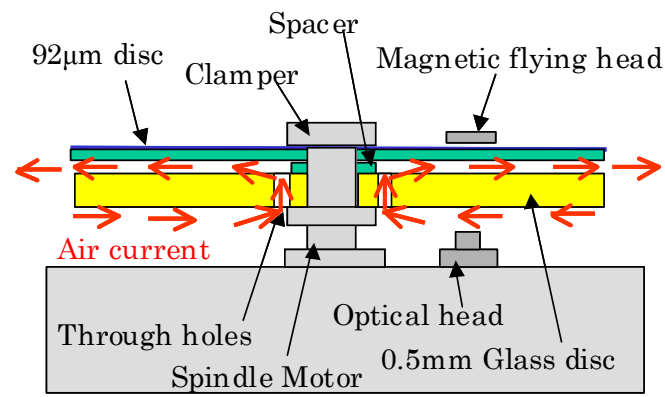

Fig. 4 shows a cross section view of the experimental setup with ADS.

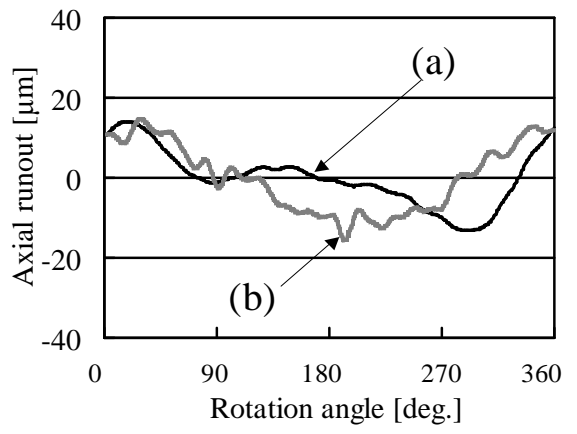

Fig. 5 Axial run out of the thin disc for one rotation at the linear velocity, $5 \mathrm{~m} / \mathrm{s}$ and the radius, $30 \mathrm{~mm}$. (a) is used ADS. (b) is used ADS without through holes on the glass substrate. 


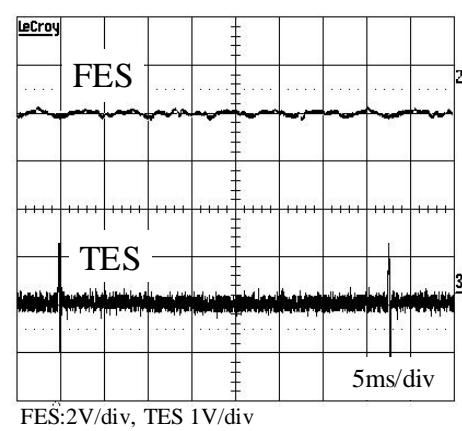

Fig. 6 FES and TES of the thin disc at the linear velocity, $5 \mathrm{~m} / \mathrm{s}$ and the radius, $30 \mathrm{~mm}$ using $\mathrm{ADS}$ with through holes on the glass substrate.

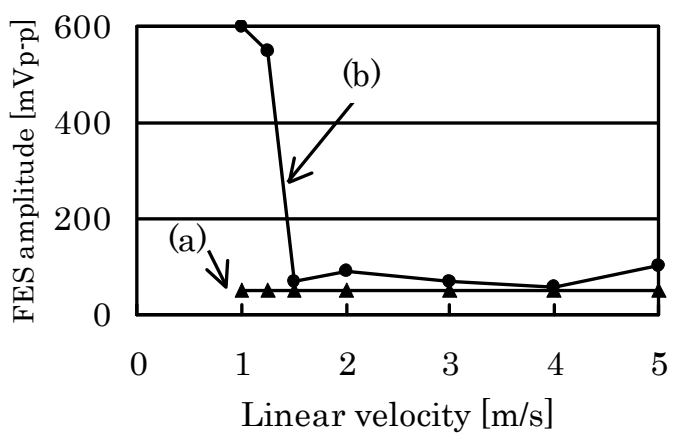

Fig. 7 Linear velocity dependence of the maximum amplitude of FES with the magnetic flying head at the radius of $30 \mathrm{~mm}$. (a) $1.2 \mathrm{~mm}$ thick disc, (b) 92 $\mu \mathrm{m}$ thick disc with the ADS.

\subsection{MO recording on thin disc}

Figure 8 shows the readout MO signal of the thin disc with the mark length of $1.0 \mu \mathrm{m}$. The signal is as clear as that of a conventional disc with the thickness of $1.2 \mathrm{~mm}$. Its carrier and noise ratio (CNR) is $45 \mathrm{~dB}$.

Figure 9 shows the mark length dependence of CNR for a conventional disc with a thickness of $1.2 \mathrm{~mm}$ and the thin disc. Both CNR slowly decrease by less than $0.5 \mu \mathrm{m}$ because of the optical limitation. The CNR of the thin disc is almost the same as the conventional disc throughout the mark length.

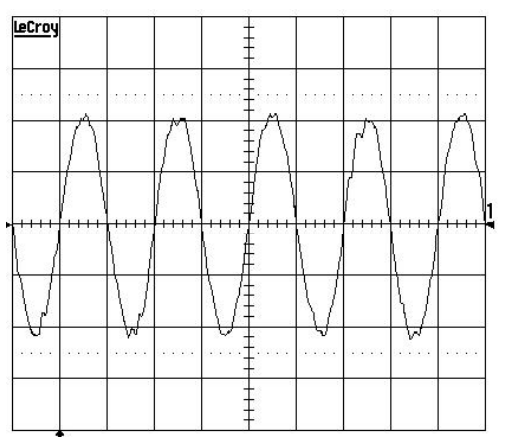

Fig. 8 Readout signal of the thin disc with the mark length of $1.0 \mu \mathrm{m}$.

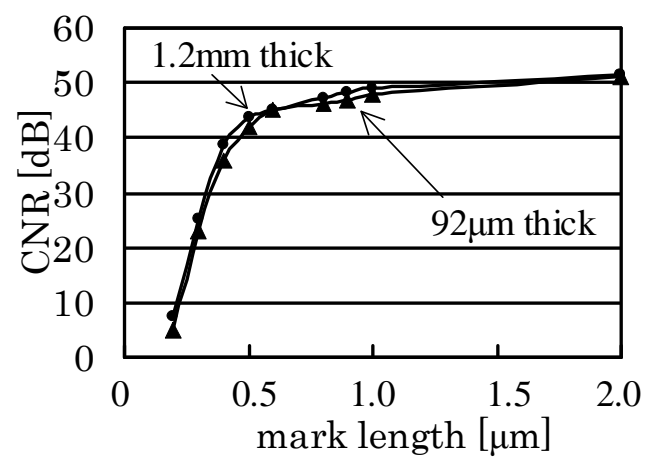

Fig. 9 Mark length dependence of CNR for conventional disc with the thickness of $1.2 \mathrm{~mm}$ and the thin disc.

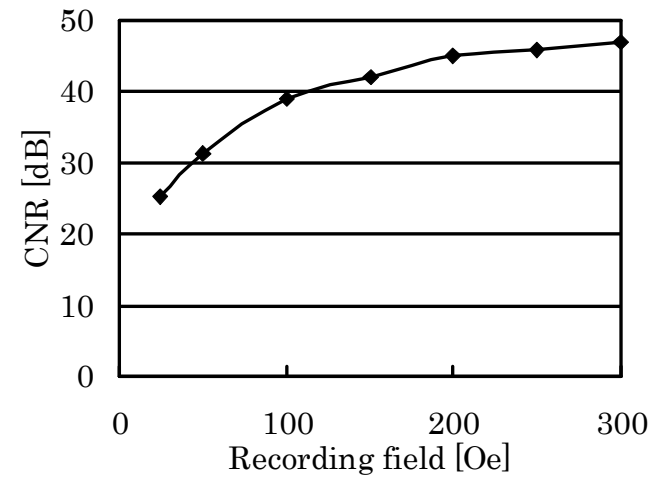

Fig. 10 Recording field dependence of $\mathrm{CNR}$ with monotone pattern for mark length of $1.0 \mu \mathrm{m}$.

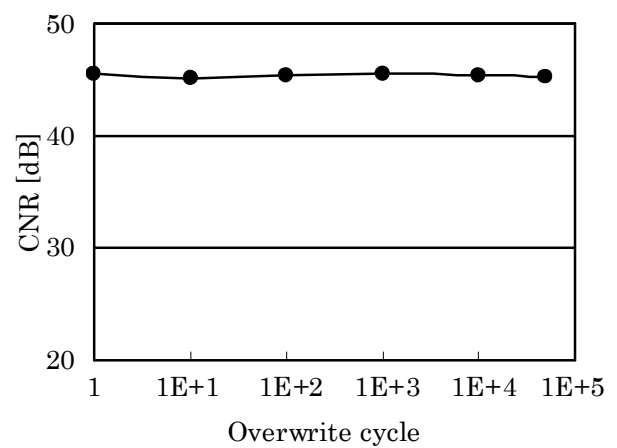

Fig. 11 CNR dependence of over write cycles for mark length of $1.0 \mu \mathrm{m}$ with the thin disc.

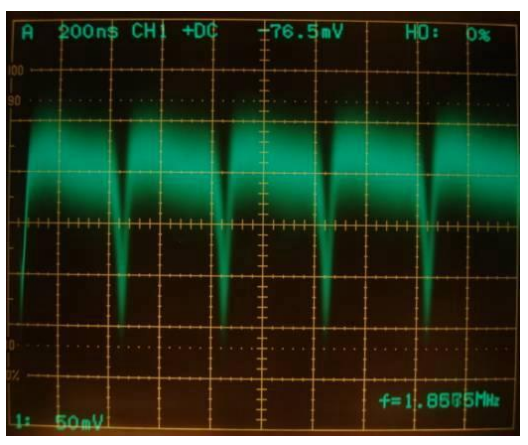

Fig. 12 Readout signal with $80 \mathrm{~nm}$ mark length on MAMMOS film with the thin disc. 
Figure 10 shows the recording field dependence of CNR with monotone pattern for mark length of $1.0 \mu \mathrm{m}$. The CNR reaches $45 \mathrm{~dB}$ above 200 Oe.

Figure 11 shows the CNR dependence of over write cycles for mark length of $1.0 \mu \mathrm{m}$ with the thin disc. After 50000 overwrites, the CNR lost only $0.5 \mathrm{~dB}$. There is no problem of overwrites.

Figure 12 shows the readout signal with $80 \mathrm{~nm}$ mark length on MAMMOS film with thin disc. The disc structure for MAMMOS is UV resin $(5 \mu \mathrm{m}) / \mathrm{Ag}$ alloy(10 $\mathrm{nm}) / \operatorname{SiN}(5 \mathrm{~nm}) / \mathrm{TbFeCo}(50 \mathrm{~nm}) / \mathrm{TbGdFe}(10 \mathrm{~nm}) /$ $\operatorname{GdFe}(20 \mathrm{~nm}) / \operatorname{SiN}(10 \mathrm{~nm}) / \mathrm{PC}$ substrate$(92 \mu \mathrm{m})$. The MAMMOS is a technology making readout possible with smaller marks under optical limitation to expand the recorded magnetic domain onto readout layer. The readout signals with $80 \mathrm{~nm}$ mark length are detected beyond optical limitation. This result indicates that thin discs are available for Magnetically induced Super Resolution such as MAMMOS.

\section{Conclusion}

We succeeded in MO recording on a thin disc with $92 \mu \mathrm{m}$ thickness using a magnetic flying head and MO pick up. The ADS was still working even when the flying head was loaded on to the thin disc. The write and read performance on TbFeCo film of the thin disc was almost the same as that of the normal disc with thickness of $1.2 \mathrm{~mm}$. The readout signals with smaller marks under the optical limitation of MO pick up were also detected with MAMMOS film on the thin disc. We can estimate that the combination of MO recording and SVOD will provide a 10 TB cartridge capacity using 100 ultra thin discs stored in a compact cartridge. Additionally, if near field recording7) with $100 \mathrm{~GB} /$ disc is adapted to SVOD, a 10 TB cartridge capacity will be realized with removability using optical memory.
Acknowledgements The authors would like to express their sincere thanks to Mr. H. Kishi, Mr. H. Yamanaka, Dr. H. Ido, Dr. H. Awano, and Dr. N. Ota for valuable discussions and for helping with the experiments.

\section{References}

1) K. Aratani, A. Fukumoto, M. Ota, M. Kanako, and K. Watanabe: Optical Data Storage '91, Proc. SPIE, 1499, 209 (1991).

2) M. Tani, M. Sekine, S. Imai, H. Awano, and N.Ohta: Optical Data Storage 2001, Tech. Dig. pp. 61-63 (2001).

3) H. Awano, H. Ido, M. Tani and Norio Ota: Int. Symp .On Optical Memory '03, Dig. We-E-02 (2003).

4) H. Awano, H. Ido, T. Iida, Y. Watanabe, H. Kishi, A. Inaba, M. Tani, H. Yamanaka, S. Osawa, M. Yoshihiro, N. Ota, T. Yoshida, T. Shimizu, I. Naniwa, Y. Abe, K. Yoshida and K. Sato: Optical Data Storage 2006, Tech. Dig. WP24 (2006).

5) T. Yoshida, T. Shimizu, I. Naniwa, Y. Abe, K. Yoshida, K. Sato, H. Awano, H. Ido, T. Iida, Y. Watanabe, H. Kishi, A. Inaba, M. Tani, H. Yamanaka, S. Osawa, M. Yoshihiro, and N. Ota: Optical Data Storage 2006, Tech. Dig. Mo-C-02 (2006).

6) M. Hennessey, D. Strand, D. Jablonski, B. Walton, B. Clark and T. Ohta,: Int. Symp. Optical. Memory and Optical Data Storage 2005, Tech. Dig. WA2 (2005).

7) A.Nakaoki, M.Shinoda, K.Saito, T.Ishimoto, T.Horigome, K.Takagi, and M.Yamamoto, $E^{* P C O S 05, ~ C a m b r i d g e, ~}$ England, Sept, (2005).

Received September 7, 2007; Revised October 10, 2007; Accepted October 24, 2007. 